\section{NAT'L INST OF STAND \& TECH R.IC

\section{NISTIR 5725}

\title{
User's Guide for RDA/SQL Validation Tests (Version 1.0)
}

\section{Kevin Brady \\ Joan Sullivan}

U.S. DEPARTMENT OF COMMERCE Technology Administration National Institute of Standards and Technology

Gaithersburg, MD 20899-0001 



\section{NISTIR 5725}

\section{User's Guide for RDA/SQL Validation Tests (Version 1.0)}

\section{Kevin Brady \\ Joan Sullivan}

U.S. DEPARTMENT OF COMMERCE

Technology Administration

National Institute of Standards and Technology

Gaithersburg, MD 20899-0001

December 1996

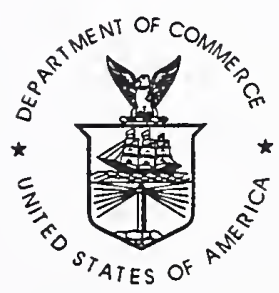

U.S. DEPARTMENT OF COMMERCE Michael Kantor, Secretary

TECHNOLOGY ADMINISTRATION

Mary L. Good, Under Secretary for Technology

NATIONAL INSTITUTE OF STANDARDS

AND TECHNOLOGY

Arati Prabhakar, Director 

User's Guide for the RDA/SQL Validation Tests (Version 1.0)

Kevin Brady

Joan Sullivan

September 1996

ABSTRACT: The RDA/SQL Validation Tests, developed by NIST, consist of a set of $C$ programs designed to test an RDA/SQL server for conformance to the international standards for Remote Database Access with an SQL Specialization. Testing is limited to the RDA Basic Application Context/Immediate Execution profile defined by the NIST OIW Stable Agreements for OSI Protocols. The validation tests use software tools provided in the public domain Iso Development Environment (ISODE) and has been operating at NIST on TCP/IP networks using the Internet Engineering Task Force (IETF) specification RFC 1006.

KEYWORDS: conformance testing; database standards; interoperability; remote database access; RDA; SQL; RFC 1006; ISODE; testing of software; validation of software

DISCLAIMER: Because of the nature of this report, it is necessary to mention vendors and commercial products. The precense or absence of a particular trade name product does not imply criticism or endorsement by the National Institute of Standards and Technology, nor does it imply that the products identified are necessarily the best available. 



\section{Table of Contents}

1. INTRODUCTION . . . . . . . . . . . . . . . . . . . . . . 1

1.1 Background . . . . . . . . . . . . . . . . . . . . 1

1.2 Scope of Testing . . . . . . . . . . . . . . . . 4

2. STRUCTURE OF RDA/SQL VALIDATION TESTS . . . . . . . . . . . 5

2.1 Description of RDA/SQL Validation Tests . . . . . . . 5

2.2 System Requirements . . . . . . . . . . . . . . . . 6

2.3 Directory Structure . . . . . . . . . . . . . . . 7

2.4 File Naming Conventions . . . . . . . . . . . . . . 7

3. INSTALLATION . . . . . . . . . . . . . . . . . . . . . . . 7

4. REPORTING . . . . . . . . . . . . . . . . . . . . . . . . . 8

5. MAINTENANCE . . . . . . . . . . . . . . . . . . . . . 8

6. REFERENCES . . . . . . . . . . . . . . . . . . . . . . . 9

APPENDIX A Sample RDA/SQL Test Program . . . . . . . . . . 11

APPENDIX B Sample Test Program Report . . . . . . . . . . 14

APPENDIX C Sample Test Program Log . . . . . . . . . . . . 15

APPENDIX D Sample Summary Report . . . . . . . . . . . . . 24 



\section{INTRODUCTION}

This user's guide describes the RDA/SQL Validation Tests and the procedures needed to test and evaluate an RDA Server implementation for conformance to international standards for Remote Database Access with an SQL Specialization (RDA/SQL).

\subsection{Background}

The purpose of the RDA specification is to standardize a method for SQI clients to access and modify the data on a remote SQL server. Many SQL products support the client/server architecture, where the user application and the SQL data server reside on separate computers. Each SQL software developer has proprietary formats and protocols for client/server communications. As a result, client and server products from different SQL software developers do not interoperate.

RDA is a standard that describes the communications protocol used between database clients and servers; i.e., what flows "over the wire". An implementation of RDA for a client application can be described as a layer of software supporting the application by formating the application's SQI requests for data into standard messages. The SQL statement itself, along with input parameter values for the SQL statement, is formatted as a structured message. The data returned to the client is then received as a structured message. The returned message could contain little more than a status code to indicate success or failure. Or, the returned message could additionally contain multiple rows of data, along with a table description (including column names, data types, precision, character set, etc.). RDA allows the client application to request the server to convert data values into data types or character sets appropriate to the client application.

An implementation of an RDA server can be described as a layer of software written on top of the SQL server. The RDA server layer of code unpacks the RDA message structure, moving the SQL statement and parameter values into local variables and then interacting with the SQL server to obtain data or to update the database. The data to be returned to the client is reformatted and restructured into an RDA message by the RDA server. Although the RDA server code could be written using a standard Embedded SQL C or CLI interface 
to an SQL database, a native (proprietary) interface to the SQL database is typically chosen by the software developer.

RDA is an international standard that specifies the functionality of a database server within a distributed open systems environment. RDA also specifies the communication service and protocol for accessing its capabilities. RDA is a two-part standard [1]. Part 1 specifies service and protocol for a generic model. Part 2 specifies the SQL specialization of the service and protocol and refers to the SQL standard [2] to define the SQL language contained in the RDA messages. The structured messages between the client and server use ASN.1 (Abstract Syntax Notation 1, defined in ISO/IEC 8824) to specify their format and the associated binary encoding rules.

RDA is positioned in the Application Layer of the Reference Model of Open Systems Interconnection (OSI) and relies on services provided by lower layers.

Because of this layered approach, RDA service requirements can also be mapped to other communications protocols, such as TCP/IP. The current NIST laboratory environment for the RDA/SQL Validation Tests uses the Internet Engineering Task Force (IETF) specification RFC 1006 to interoperate with other RDA/SQL clients and servers over the Internet. Figure 1 shows the RDA service layers for client/server architectures, although, in theory, an RDA client or service product could support both TCP/IP and OSI protocols, it is probable that each product will support only one of the protocols. 


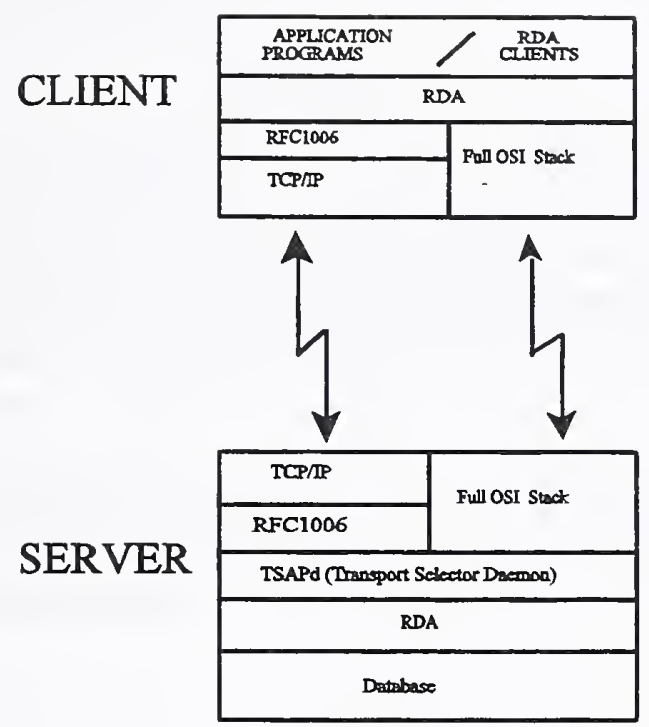

Figure 1. RDA Service Layers for Client/Server Architectures

Although a thorough test of the database server's support for the SQL language is also needed, this set of tests does not attempt that level of testing. Instead, a separate evaluation of the SQL server should be conducted using the NIST SQI Test Suite [3].

The NIST RDA/SQL laboratory environment in which the validation tests were developed is part of an RDA testbed announced in the February 8, 1994, Federal Register. As a result of research into RDA by NIST and other organizations, additional prototype tools and demonstration software have been developed:

distributed query processor

GUI monitor for distributed query processor

RDA server for Informix

RDA server for Oracle

RDA server for Sybase

RDA sniffer

RDA GUI client

RDA applications

The distributed query processor and GUI monitor, as well as RDA 
servers for SQL products (Informix, Oracle, and Sybase), are enhancements of software donated by NRaD (Naval Ocean Surveillance $R \& D, \operatorname{san}$ Diego, CA).

\subsection{Scope of Testing}

All of the test programs validate standard behavior of an RDA server with access to an SQL database; $i . e .$, the RDA server layer of code is not tested in stand-alone mode. Also, an RDA client product that may be sold with the RDA server, is not tested by these programs.

Fourteen different message types are defined in the RDA standard to provide five services. These services and messages are:

Dialogue Management services (to start and end RDA dialogues)

Initialize

Synchronize

Terminate

Transaction Management services (to start and end RDA transactions)

Begin Transaction

Commit

Rollback

Control services (to report the status or cancel existing operations)

Cancel

Status

Resource Handling services (to enable or disable access by RDA clients to data resources)

Open

Close

Database language services (to access and modify data resources)

Execute DBL (immediate execution)

Define DBL

Invoke DBL

Drop DBI

Two application contexts are defined for RDA dialogues, depending on whether or not the application requires distributed transactions (transactions spanning two or more servers). Transaction processing services (TP), invoking two-phase commit protocols, are 
defined in a separate $T P$ standard [4]. The two application contexts are called:

RDA Basic application-context

RDA TP application-context

Testing is limited to a minimal profile, selecting from the above options only those needed for the simplest access to the full SQL language. The profile selected for testing was defined cooperatively in the NIST Open Systems Environment (OSE) Implementors Workshops (OIW) Stable Implementation Agreements for OSI Protocols [5]. The profile used in RDA/SQL testing is called "Immediate Execution." This profile requires the following functional units: dialogue initialization, dialogue termination, transaction management, resource handling, and immediate execution DBL. Testing is also restricted to RDA Basic Application Context. The OIW RDA agreements provide additional restrictions and specify min-max limits.

Although the RDA standards and the OIW agreements specify access over OSI networks, the RDA/SQL Validation Tests were developed in a TCP/IP laboratory environment and remote testing of products was accomplished over the Internet. In theory, all the software developed by NIST for RDA/SQL testing can be deployed in a strict OSI environment after minor configuration steps.

The SQI/RDA test programs are not comprehensive, due to limited resources. The goal of the set of tests is to ensure that straight-forward SQL access will be successful. The focus of testing has been on exercising the options of the Execute DBL message type (which carries the SQL request and the SQL data) and verifying support for min-max limits. Only modest effort was expended on testing other message types and error conditions.

\section{STRUCTURE OF RDA/SQL VALIDATION TESTS}

\subsection{Description of RDA/SQL Validation Tests}

The RDA/SQL Validation Tests are a collection of 74 C-language programs, each testing a specific feature or requirement of the RDA standard. The test programs make liberal use of a library of subroutines, provided in a separate directory. The testbed of tables and data used by the test programs is created by an 
initialization program containing SQL statements to create tables and insert rows. Since test programs may alter the testbed, there is another program to assist in restoring the testbed to its initial state. This program simply deletes SQL objects, in order to allow the initialization program to rerun cleanly. Reporting for the RDA/SQL Validation Tests consists of capturing the "PASS" or "FAIL" lines from the program logs and combining these lines into a summary printout.

The RDA/SQL Validation Tests were developed using ISODE tools. Softcopy of the RDA SQL Specialization ASE ASN.I Module, defined on page 35 of the standard ISO/IEC 9579-2:1993, was used by the ISODE tools to generate $\mathrm{C}$-language header files and encoding/decoding subroutines. These subroutines are used by the test programs. ISODE tools also provide the RFC 1006 communications mapping software needed to run over TCP/IP protocols in the NIST laboratory and over the Internet. In order to execute the RDA/SQL Validation Tests, it is necessary to install the ISODE tools first.

Makefiles and scripts to assist in creating and running the test executables in a Unix environment are provided with the validation tests. For non-Unix platforms, the tester will need to create similar makefiles and scripts. Since Unix platforms are widely available, we recommend installing the validation tests (the $\mathrm{RDA} / \mathrm{SQL}$ client) on a Unix platform. Keep in mind that the RDA/SQL client and the RDA/SQL server to be tested do NOT need to be compatible platforms. They need have nothing in common except the ability to support the RDA/SQL protocol over TCP/IP (or some other communications protocol).

\subsection{System Requirements}

In order to install and execute the validation tests successfully, the following are suggested:

- ISO conforming RDA server (on the server side)

- ANSI/ISO conforming SQL database (on the server side)

- ISODE Version 8.0 (installed on the client side)

- ANSI/ISO conforming C compiler (on the client side)

Since the RDA/SQI Validation Tests are designed to exercise the features of the RDA server rather than the other components above, a C compiler or SQL database which is not fully conforming may 
still be adequate.

\subsection{Directory Structure}

The files are organized in two directories. The top directory is called CONF (for conformance testing), and it contains only one subdirectory, LIB. Directory CONF contains all the test programs, a Makefile, and a header file (testing.h) for the test programs. The subdirectory LIB contains all the support subroutines called by the test programs to initialize, terminate, encode, decode, build customized messages, check return values, etc.

\subsection{File Naming Conventions}

The following are naming conventions used in directories CONF and LIB :

RDAxxx.c a test program

RDAxxx.rpt a summary of the log created by the program

RDAxxx.log the log created by executing the program

Please refer to the appendicies for examples as follows:

Appendix A Sample RDA/SQL test Program

Appendix B Sample Test Program Report

Appendix C Sample Test Program Log

Makefiles, subroutines, and header files follow $\mathrm{C}$-language naming conventions. The program to initialize the SQL tables and data is called CREATE.C. The program to drop tables prior to a clean restart is called DROP.C. The text file README.out is documentation of the test specifications for the test programs. README.out is generated by program build_readme.c which extracts documentation coded as comments in the individual test programs.

\section{INSTALLATION}

The RDA/SQL Validation Tests software is publicly available over the World wide web from the URL http://www.nist.gov/itl/div897/ctg/rda_form.htm

The test software is a compressed Unix TAR archive. Since the test software is built using ISODE tools, the ISODE compressed Unix TAR archive should also be downloaded from the same site. After downloading, uncompressing, and extracting from the TAR archives, 
the next step is to build the test programs.

Install the ISODE tools according to the instructions provided.

There are two files that need to be edited to identify the RDA server(s) to be tested. File isoentities (in the root directory of ISODE) must specify P-selector, S-selector, T-selector, IP address and (optional) port. File load.c (in directory LIB) must specify host name (IP address) and service.

Review the Makefiles in directories LIB and CONF to point to the ISODE library header files. In directory LIB, type "make" to create the C-language library file libconf.a. In the directory CONF, type "make" to create all the $C$ test programs and to execute them in the correct order. Typically, this order is DROP and CREATE, followed by all of the test programs in alphabetical order. Program REPORT is run last to summarize the results from executing the test programs. For additional information about the results of an individual program, read the ".log" and ".rpt" text files created by the test program.

To make a single executable, e.g., RDA001, type "make RDA001". To execute a single executable, e.g., RDA001, type "RDA001 hostname", where hostname matches one of the host names in file load.c.

\section{REPORTING}

Program REPORT summarizes the results in the log files. The test programs are listed in ascending order with a result value of pass, fail, missing, or aborted. Subtotals for each category are printed at the end of the report. See Appendix D for a sample report.

Program REPORT reads the README.out file to obtain one-line descriptions for the test programs, so program build_readme should be run prior to program REPORT. The REPORT output file name is REPORT.log.

\section{MAINTENANCE}

Although the test programs have been debugged and executed on several RDA servers, errors in interpretation or in coding are 
possible. Questions about the RDA/SQL Validation Tests should be directed to:

Joan M. Sullivan

National Institute of Standards and Technology

Bldg. 820, Room 562

Gaithersburg, MD 20899

phone: (301) 975-3258

FAX: (301) $948-6213$

joan.sullivan@nist.gov

Maintenance of corrected or enhanced programs will consist of replacing the original program in the online Unix TAR archive. A maintenance log, containing the date and description of each modification, will be available online in the file named update. $\log$.

\section{REFERENCES}

[1] ISO/IEC 9579. Open Systems Interconnection - Remote Database Access (RDA), Part 1: Generic Model and Part 2: SQL Specialization, International Standard IOS/IEC 9579:1993, American National Standard ANSI/ISO/IEC 9579:1993, American National Standards Institute, New York, NY 10036, December 1993.

[2] ISO/IEC 9075. Database Language SQL, International Standard ISO/IEC 9075:1992, American National Standard X3.135-1992, American National Standards Institute, New York, NY 10036, November 1992 .

[3] NIST OIW. Stable Implementation Agreements for OSI Protocols, Version 6, Edition 1, NIST Open Systems Environment Workshop, document NIST SP 500-206, December 1992. Part 19 - Remote Database Access.

[4] NIST SQL Test Suite validation procedures online at URL ftp://speckle.ncsl.nist.gov/sql-testing/PrevalPaperwork/ procedur.val

[5] ISO/IEC 10026. Distributed Transaction Processing (TP) - Part 
1: Model, Part 2: Service Definition, and Part 3: Protocol Specification (to be published).

[6] SQL Environments, Federal Information Processing Standards Publication, FIPS PUB 193, U.S. Department of Commerce, National Institute of Standards and Technology, February 3, 1995. 


\begin{abstract}
APPENDIX A Sample RDA/SQL Test Program
\#include "testing.h"

char *name $=$ "RDA025";

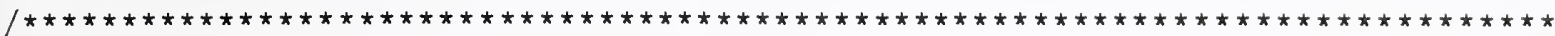

4.1.7.1.1 R-ExecuteDBL Service

NOTE: The sniffer will wait on an $r$-ExecuteDBL-RC for each $r-$ ExecuteDBL-RI sent to the server. The server "fails" if it does not respond within the timeout period (some large default value) and tester does not justify failure to respond (e.g., network went down).

0025 CT 4 SQLDBLArgumentValues $\rightarrow 4$ sQLDBExceptions, DELETE

! DELETE FROM RX

WHERE DRUG_ID $=: H$ AND PAYMENT_METHOD $=: H$

in: [ArgVal:

$\begin{array}{ll}7444 & \text { 'cash' } \\ 7444 & \text { ' } \mathrm{x} \times \times \times \\ 0000 & \text { 'cash' } \\ 7004 & \text { 'insu'] }\end{array}$

out : [SQLCODE/SQLSTATE :

o '00000'

$100 ' 02000^{\prime}$

100 '02000'

0 100000, ]

ROLLBACK WORK

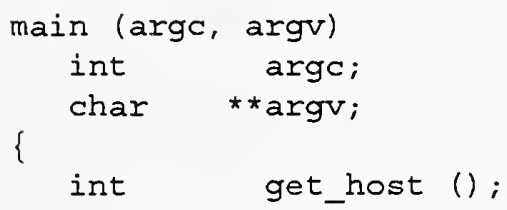

fill_initialize_ri () ; 
strcpy (APDU_0.UserAuData, username);

strcpy (APDU_o.IdeofUser, password);

encode_initiāize_ri () ;

delay (CA);

check_errors ("Initialize-request");

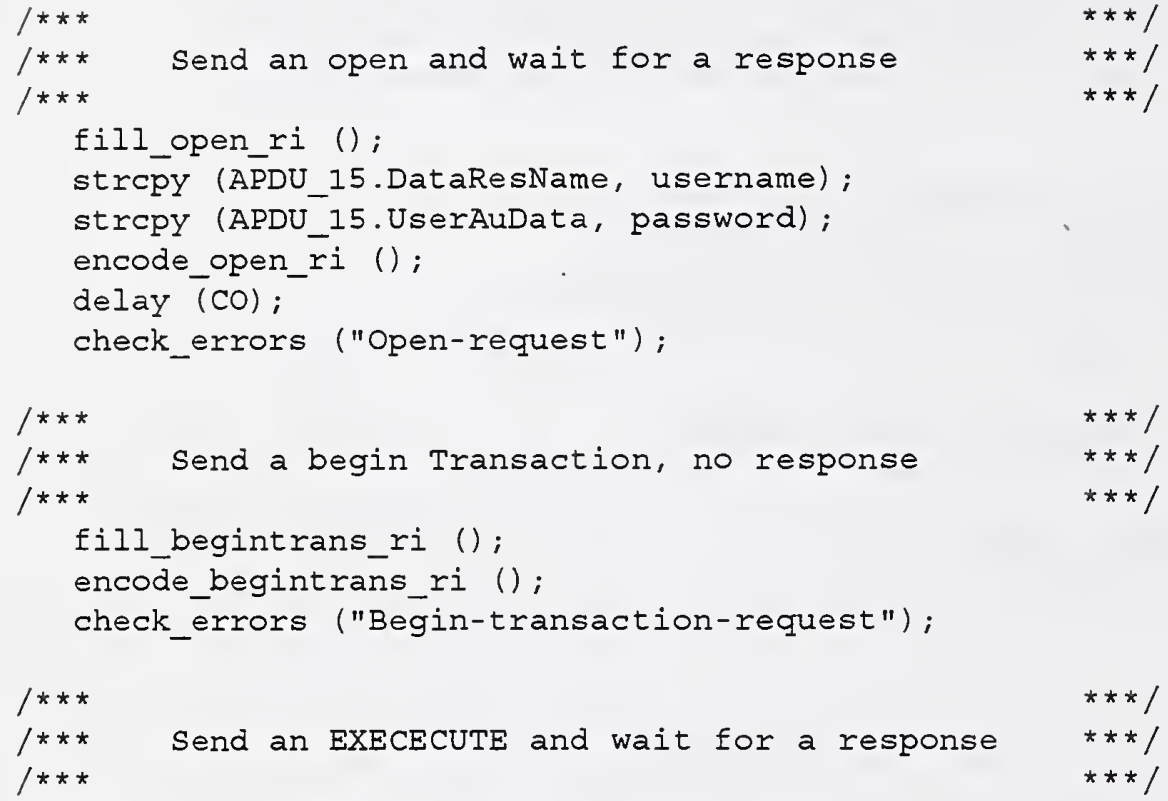




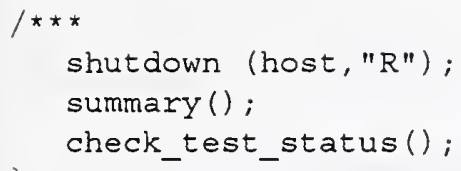




\section{APPENDIX B Sample Test Program Report}

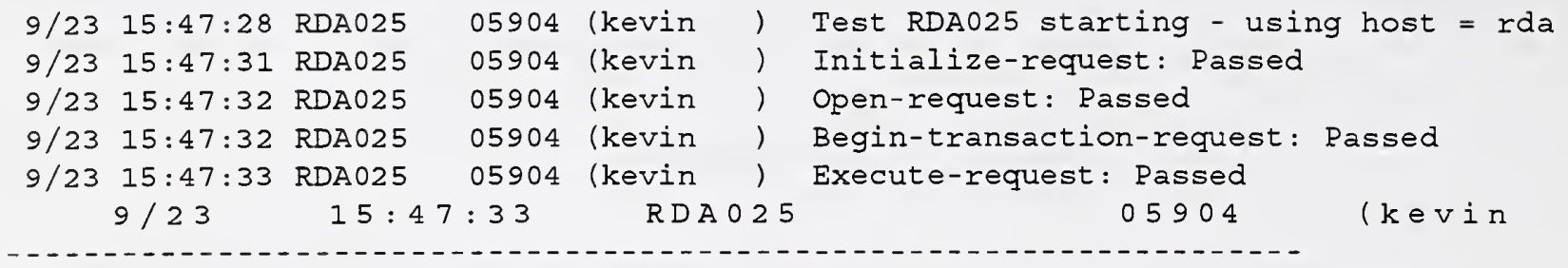

9/23 15:47:33 RDA025 05904 (kevin) ) - - - . - . - . - .
$9 / 23$
$15: 47: 33$
RDAO2 5

05904

( $k$ e vin

$9 / 23 \quad 15: 47: 33$ RDA025

$9 / 23 \quad 15: 47: 33$ RDA025

$9 / 23 \quad 15: 47: 33$ RDA025

$9 / 23 \quad 15: 47: 33$ RDA025

9/23 15:47:33 RDA025

$9 / 23 \quad 15: 47: 33$ RDA025

$9 / 23 \quad 15: 47: 33$ RDA025

$9 / 23 \quad 15: 47: 33$ RDA025

$9 / 23 \quad 15: 47: 33$ RDA025

$9 / 23 \quad 15: 47: 33$ RDA025

$9 / 23 \quad 15: 47: 33$ RDA025

$9 / 23 \quad 15: 47: 33$ RDA025

$9 / 23 \quad 15: 47: 33$ RDA025

$9 / 23 \quad 15: 47: 33$ RDA025

$9 / 23 \quad 15: 47: 33$ RDA025

$9 / 23 \quad 15: 47: 33$ RDA025

$9 / 23 \quad 15: 47: 33$ RDA025

$9 / 23 \quad 15: 47: 33$ RDA025

$9 / 23 \quad 15: 47: 33$ RDA025

$9 / 23 \quad 15: 47: 33$ RDA025

$9 / 23 \quad 15: 47: 33$ RDA025

$9 / 23 \quad 15: 47: 33 \quad$ RDA025

$9 / 23 \quad 15: 47: 33 \quad \mathrm{RDA} 025$

$9 / 23 \quad 15: 47: 33$ RDA025

$9 / 23 \quad 15: 47: 33$ RDA025

$9 / 23 \quad 15: 47: 33$ RDA025

$9 / 23 \quad 15: 47: 33$ RDA025

9/23 15:47:33 RDA025

9/23 15:47:33 RDA025

9/23 15:47:33 RDA025

$9 / 23 \quad 15: 47: 33$ RDA025

\begin{tabular}{|c|c|}
\hline 5904 & (kevin \\
\hline 05904 & (kevin \\
\hline 05904 & (kevin \\
\hline 05904 & (kevin \\
\hline 05904 & (kevin \\
\hline 05904 & (kevin \\
\hline 05904 & (kevin \\
\hline 05904 & (kevin \\
\hline 05904 & (kevin \\
\hline 05904 & (kevin \\
\hline 05904 & (kevin \\
\hline 05904 & (kevin \\
\hline 05904 & (kevin \\
\hline 05904 & (kevin \\
\hline 05904 & vin \\
\hline 05904 & vin \\
\hline 05904 & (kevj \\
\hline 05904 & (kevin \\
\hline 05904 & (kevin \\
\hline 05904 & (kevin \\
\hline 05904 & (kevin \\
\hline 05904 & (kevin \\
\hline 05904 & vin \\
\hline 05904 & (k \\
\hline 05904 & (kevin \\
\hline 05904 & (kevin \\
\hline 05904 & (kevi \\
\hline 05904 & (kevin \\
\hline 05904 & (k \\
\hline 05904 & \\
\hline 05904 & \\
\hline
\end{tabular}

Checked 1 Initialize Requests found 0 bad. Checked 1 Initialize confirms found 0 bad. Checked 0 syncronize Requests found 0 bad. Checked 1 Terminate Requests found 0 bad. Checked 1 Terminate confirms found 0 bad. Checked 1 Begintrans Requests found 0 bad. Checked 0 Begintrans Confirms found 0 bad. Checked 0 commit Requests found 0 bad. checked 0 commit Confirms found $0 \mathrm{bad}$. Checked 1 Rollback Requests found 0 bad. Checked 1 Rollback Confirms found 0 bad. Checked 0 Cancel Checked 0 Cancel Checked 0 Status Checked 0 Status Checked 1 Open Checked 1 Open Checked 1 close Checked 1 close Checked 1 Execute Checked 1 Execute Checked o Define Checked o Define Checked o Invoke Checked o Invoke Checked 0 Drop Checked 0 Drop Requests found $0 \mathrm{bad}$. Confirms found 0 bad. Requests found 0 bad. Confirms found 0 bad. Requests found $0 \mathrm{bad}$. Confirms found 0 bad. Requests found $0 \mathrm{bad}$. Confirms found 0 bad. Requests found 0 bad. Confirms found $0 \mathrm{bad}$. Requests found 0 bad. Confirms found 0 bad. Requests found 0 bad. Confirms found 0 bad. Requests found $0 \mathrm{bad}$. confirms found 0 bad. Test RDA025 Complete.

RDA025 : Completion Status: PASSED. 


\section{APPENDIX C Sample Test Program Log}

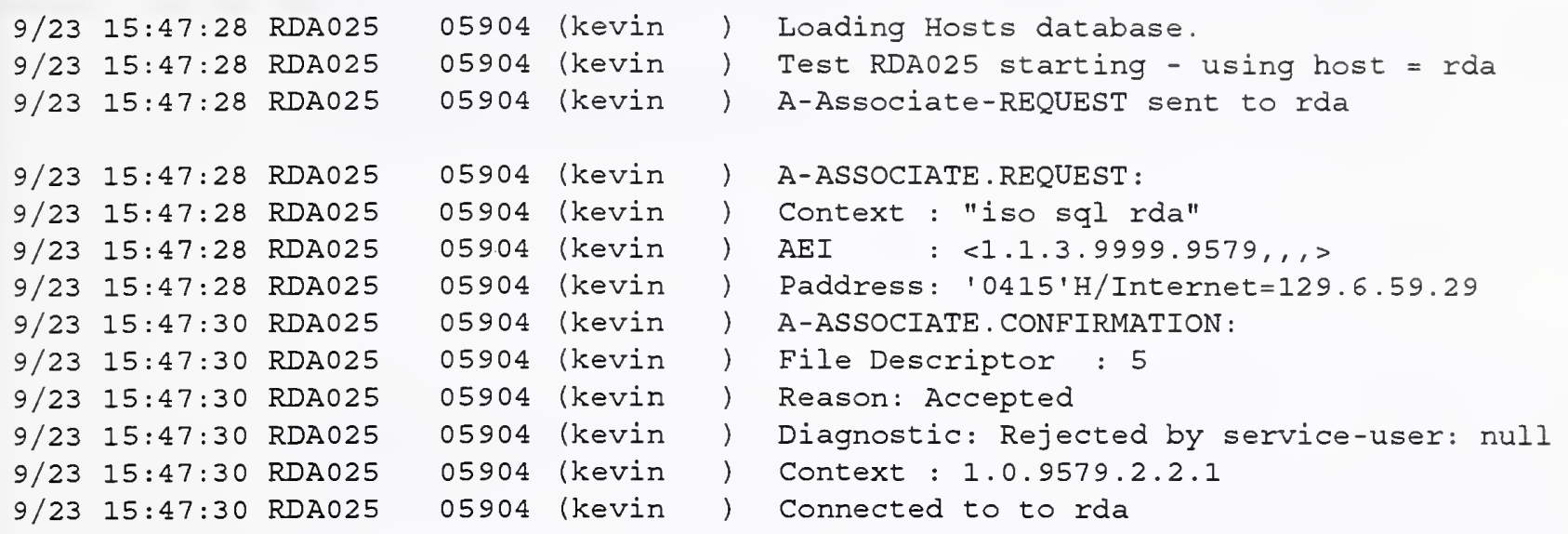




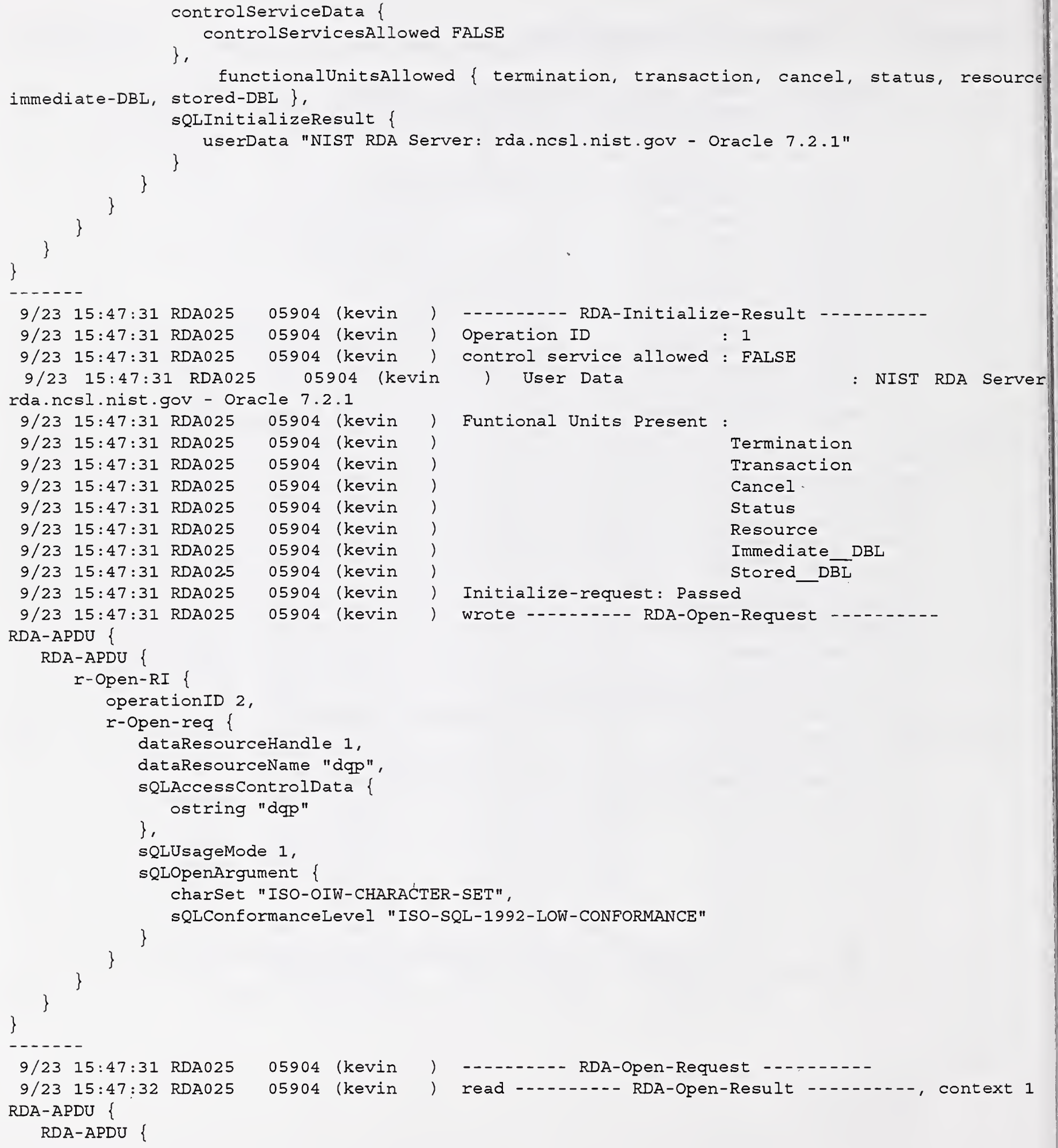




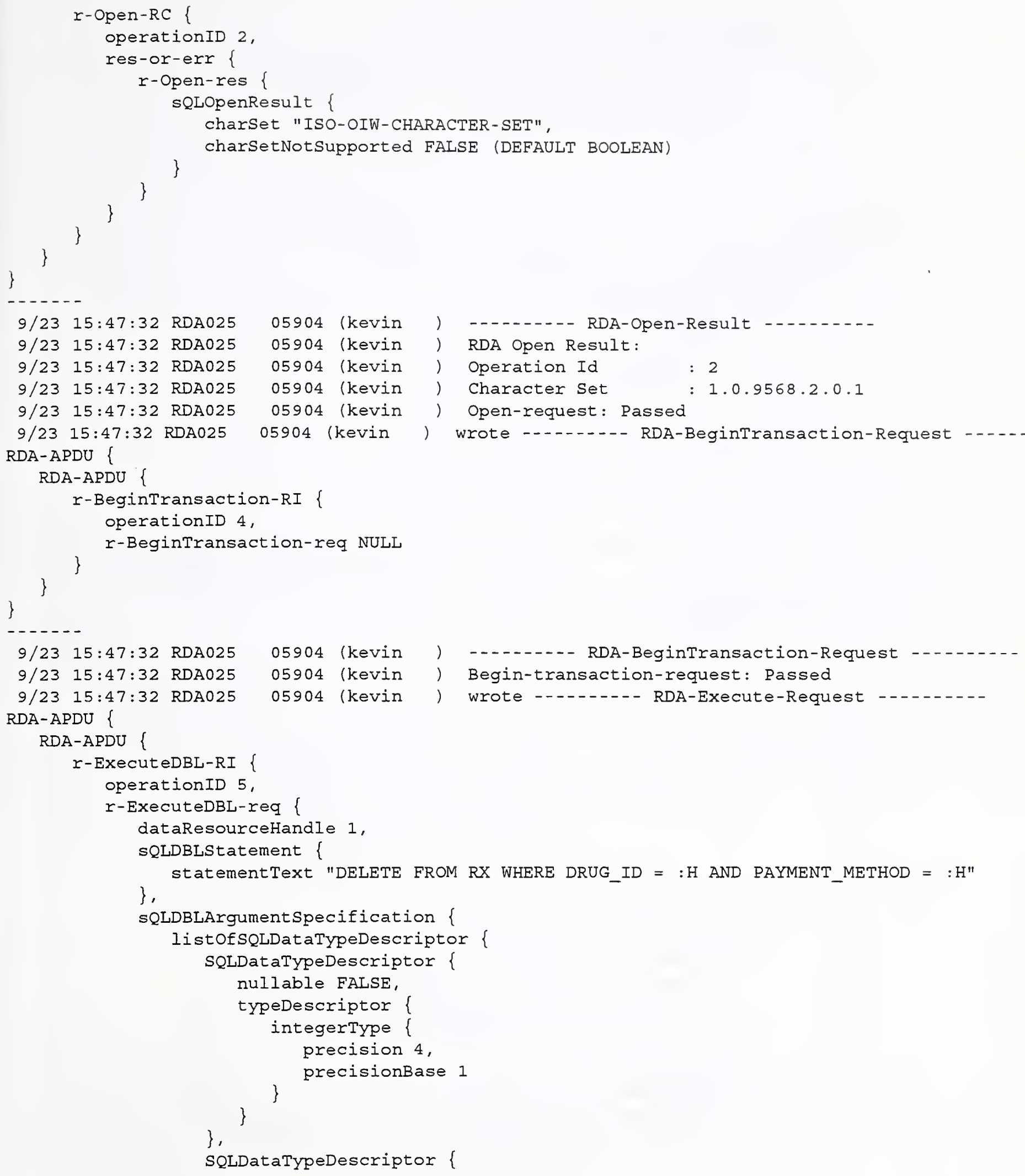




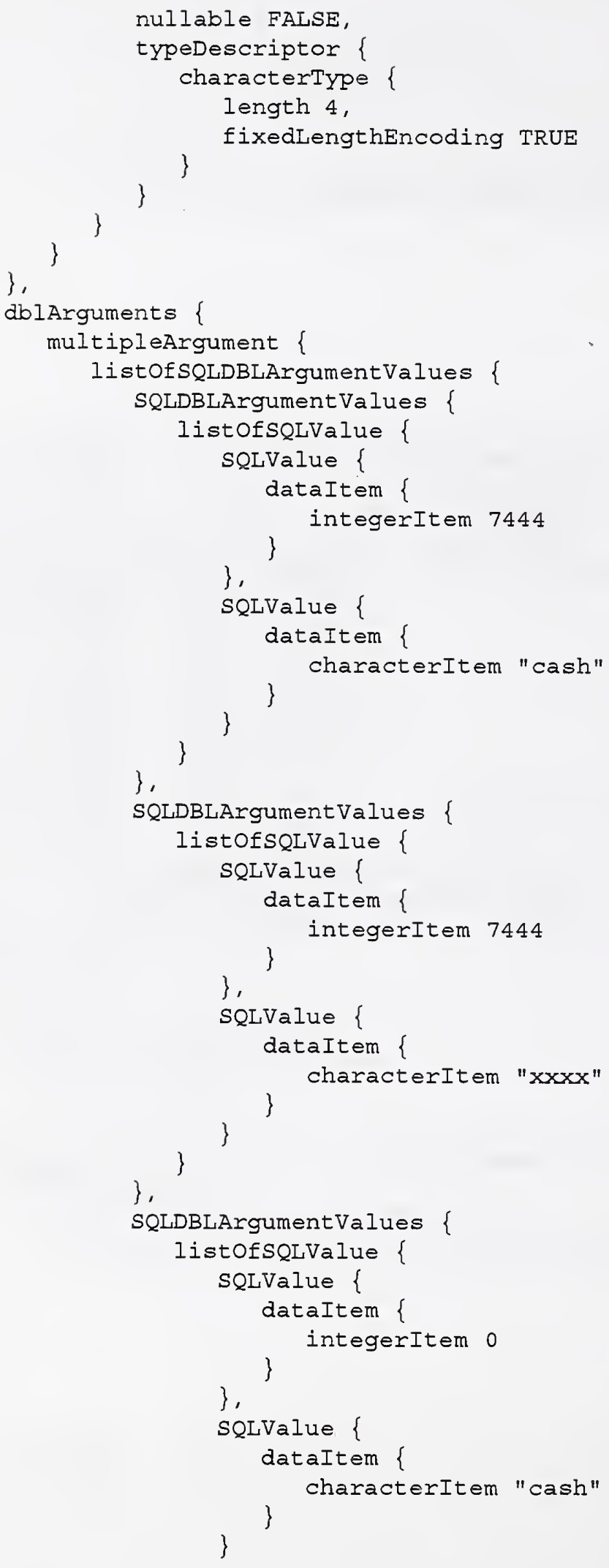




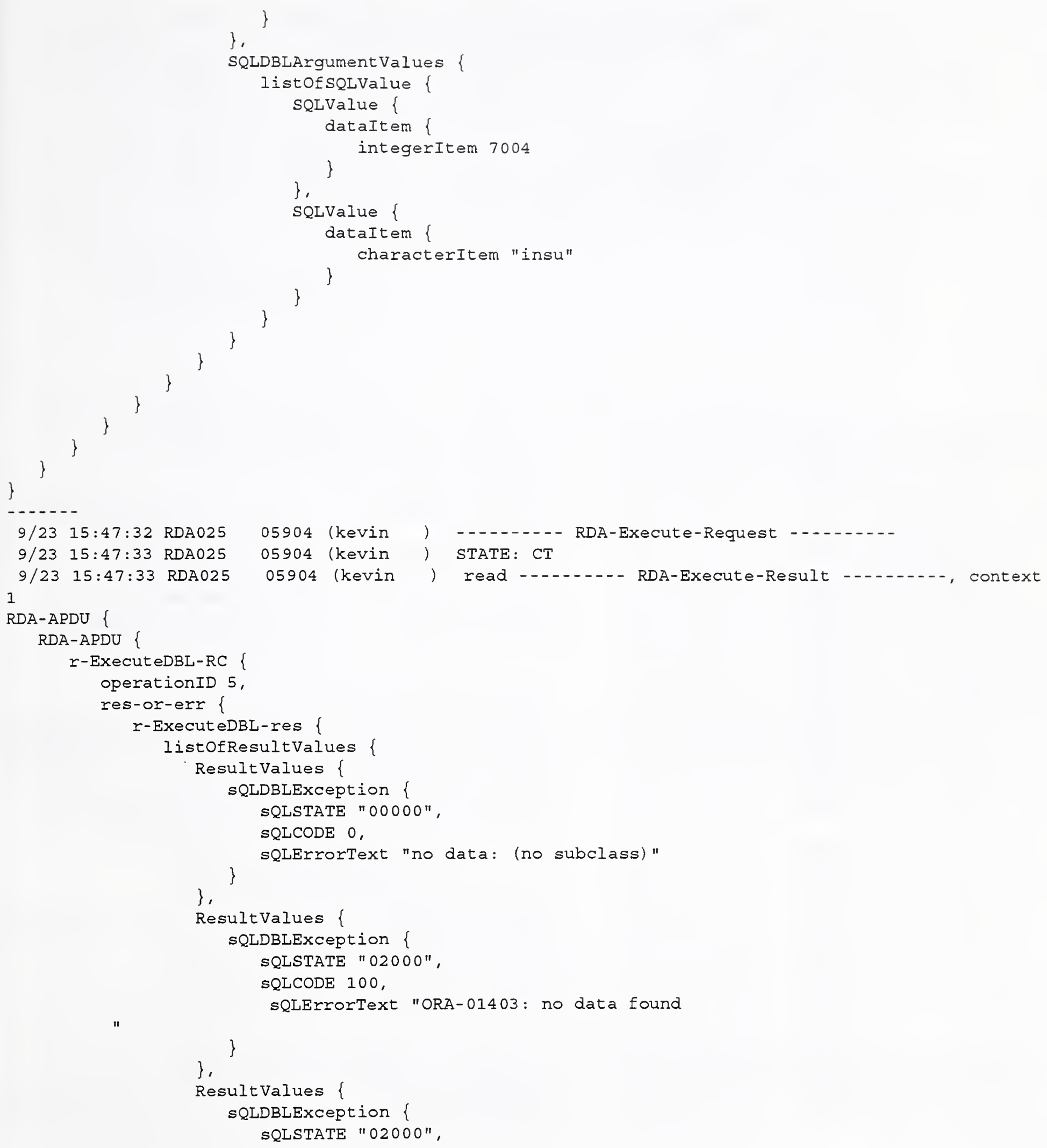




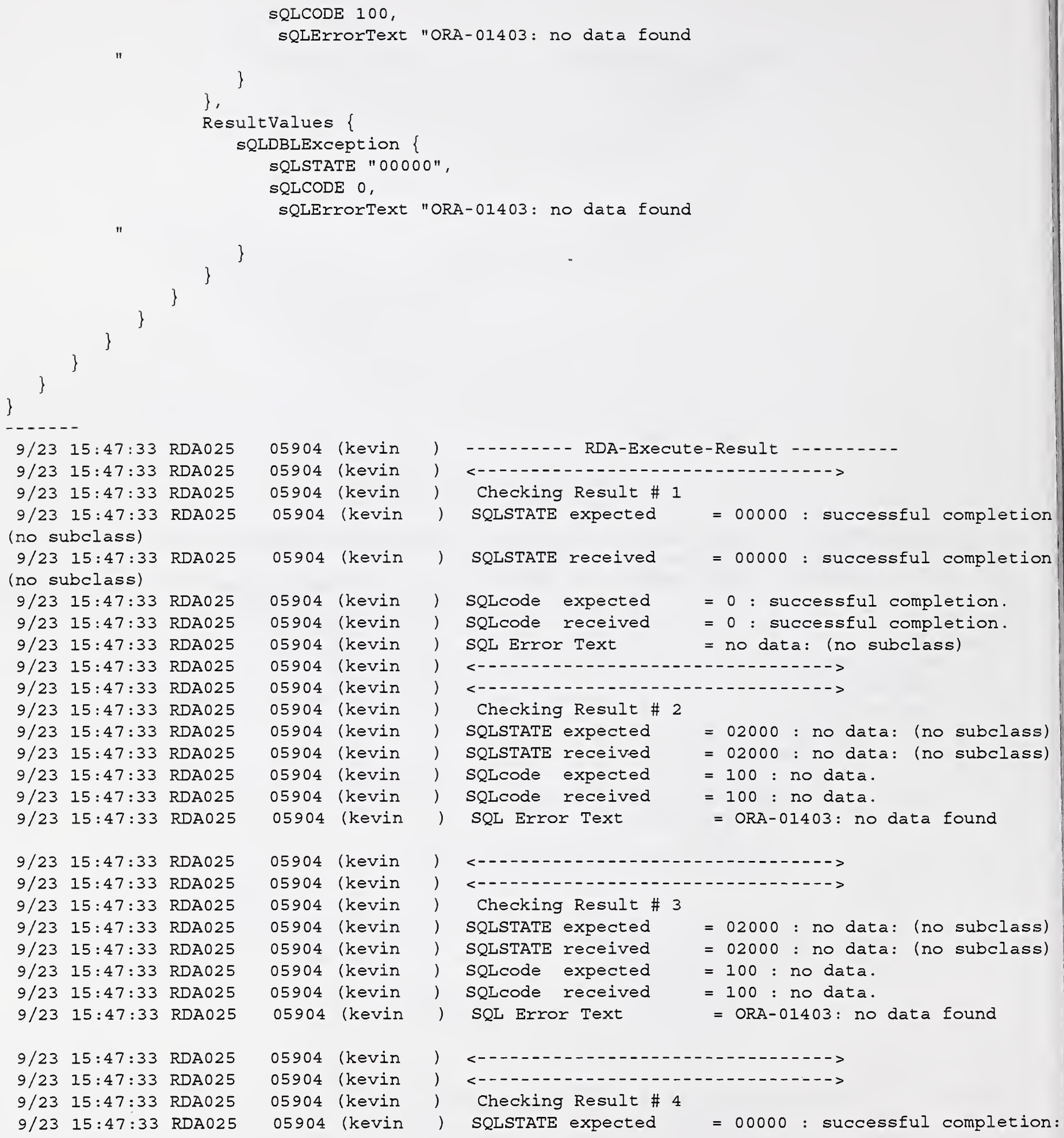




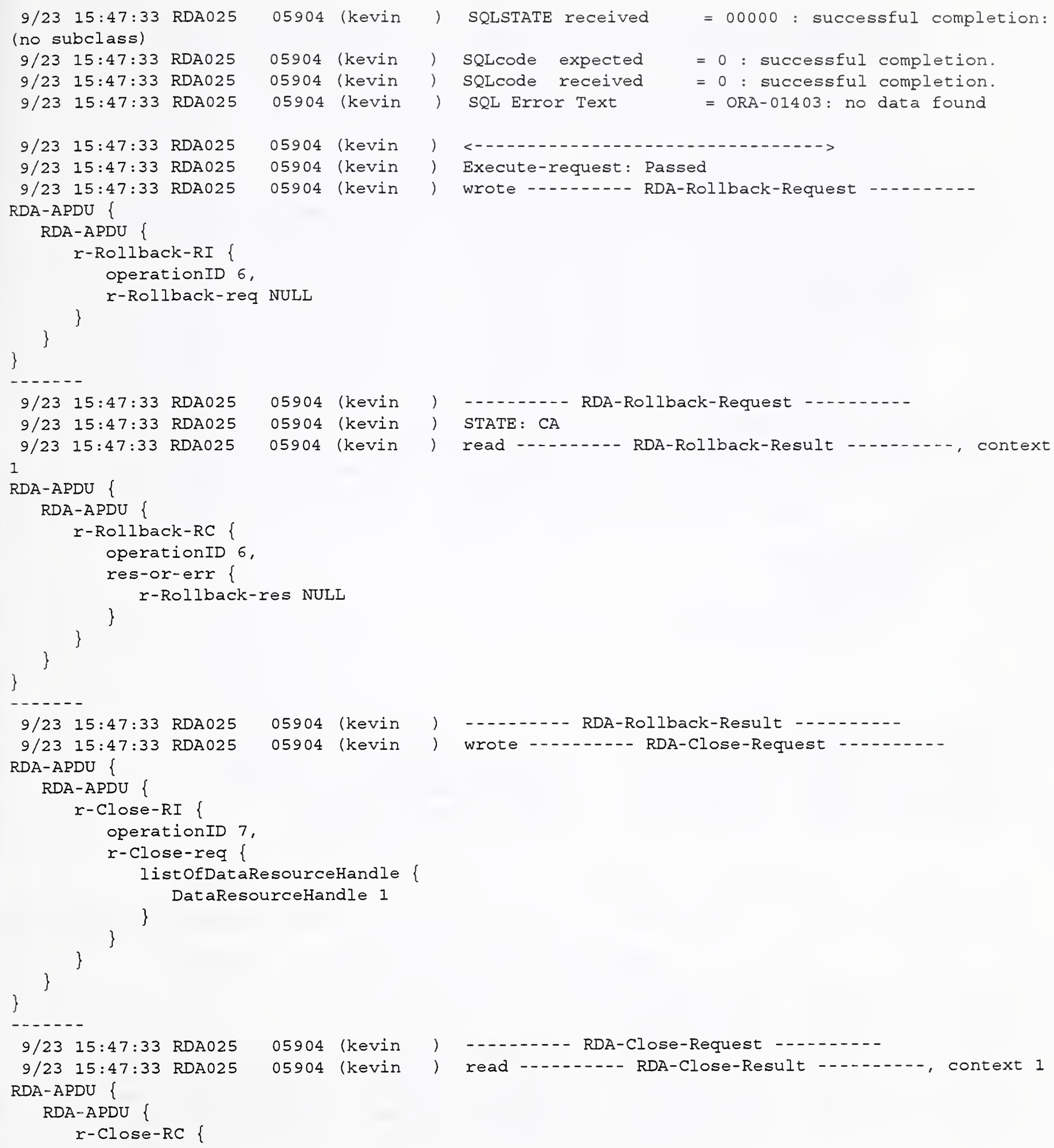




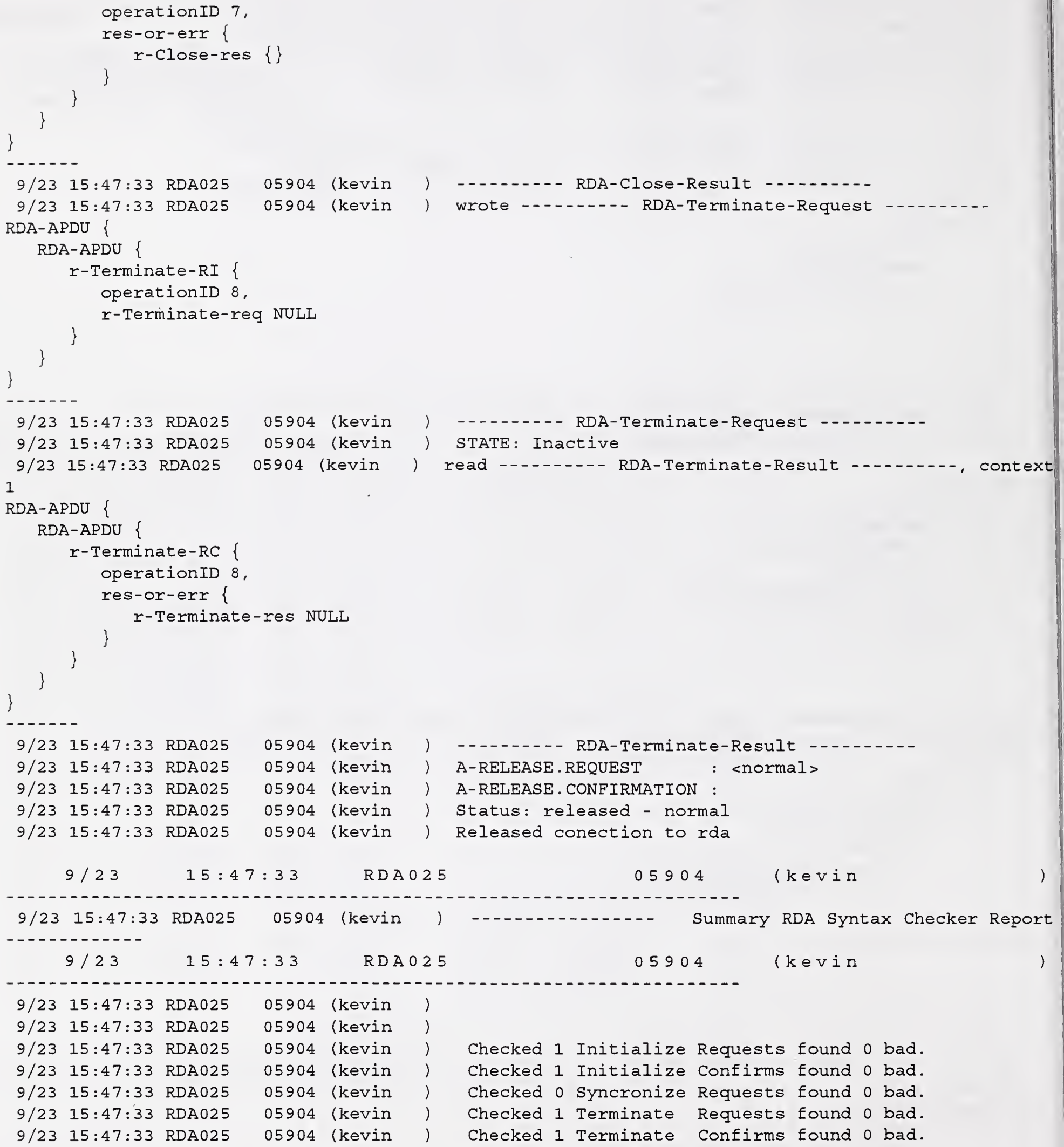

$9 / 23$

$15: 47: 33$

RDAO2 5

05904

( $k$ e vin

$9 / 2315: 47: 33$ RDA025 05904 (kevin) )

$9 / 23$

$15: 47: 33$

RDAO2 5

05904

( k e vin

$9 / 23 \quad 15: 47: 33$ RDA025

$9 / 23 \quad 15: 47: 33$ RDA025

$9 / 23 \quad 15: 47: 33$ RDA025

$9 / 23 \quad 15: 47: 33$ RDA025

9/23 15:47:33 RDA025

$9 / 23 \quad 15: 47: 33$ RDA025

$9 / 23 \quad 15: 47: 33$ RDA025

05904 (kevin ) 05904 (kevin ) 05904 (kevin ) 05904 (kevin ) 05904 (kevin ) 05904 (kevin ) 05904 (kevin )

Checked 1 Initialize Requests found 0 bad. Checked 1 Initialize confirms found 0 bad. Checked 0 Syncronize Requests found 0 bad. Checked 1 Terminate Requests found 0 bad. Checked 1 Terminate confirms found 0 bad. 


\begin{tabular}{|c|c|c|c|c|}
\hline $9 / 23$ & $15: 47: 33$ & $\mathrm{RDA0} 25$ & 05904 & (kevin \\
\hline $9 / 23$ & $15: 47: 33$ & $\mathrm{RDA} 025$ & 05904 & (kevin \\
\hline $9 / 23$ & $15: 47: 33$ & $\mathrm{RDA} 025$ & 05904 & (kevin \\
\hline $9 / 23$ & $15: 47: 33$ & $\mathrm{RDA} 025$ & 05904 & (kevin \\
\hline $9 / 23$ & $15: 47: 33$ & $\mathrm{RDA} 025$ & 05904 & (kevin \\
\hline $9 / 23$ & $15: 47: 33$ & $\mathrm{RDA} 025$ & 05904 & (kevin \\
\hline $9 / 23$ & $15: 47: 33$ & $\mathrm{RDA} 025$ & 05904 & (kevin \\
\hline $9 / 23$ & $15: 47: 33$ & $\mathrm{RDA} 025$ & 05904 & (kevin \\
\hline $9 / 23$ & $15: 47: 33$ & $\mathrm{RDA} 025$ & 05904 & (kevin \\
\hline $9 / 23$ & $15: 47: 33$ & RDA025 & 05904 & (kevin \\
\hline $9 / 23$ & $15: 47: 33$ & RDA025 & 05904 & (kevin \\
\hline $9 / 23$ & $15: 47: 33$ & RDA025 & 05904 & (kevin \\
\hline $9 / 23$ & $15: 47: 33$ & $\mathrm{RDA} 025$ & 05904 & (kevin \\
\hline $9 / 23$ & $15: 47: 33$ & $\mathrm{RDA} 025$ & 05904 & (kevin \\
\hline $9 / 23$ & $15: 47: 33$ & $\mathrm{RDA} 025$ & 05904 & (kevin \\
\hline $9 / 23$ & $15: 47: 33$ & RDA025 & 05904 & (kevin \\
\hline $9 / 23$ & $15: 47: 33$ & RDA025 & 05904 & (kevin \\
\hline $9 / 23$ & $15: 47: 33$ & $\mathrm{RDA} 025$ & 05904 & (kevin \\
\hline $9 / 23$ & $15: 47: 33$ & $\mathrm{RDA} 025$ & 05904 & (kevin \\
\hline $9 / 23$ & $15: 47: 33$ & RDA025 & 05904 & (kevin \\
\hline $9 / 23$ & $15: 47: 33$ & RDA025 & 05904 & (kevin \\
\hline $9 / 23$ & $15: 47: 33$ & $\mathrm{RDAO} 25$ & 05904 & (kevin \\
\hline $9 / 23$ & $15: 47: 33$ & RDA025 & 05904 & (kevin \\
\hline $9 / 23$ & $15: 47: 33$ & RDA025 & 05904 & (kevin \\
\hline
\end{tabular}

Checked 1 Begintrans Requests found 0 bad. Checked 0 Begintrans confirms found 0 bad. checked 0 commit Requests found 0 bad. checked 0 commit Confirms found 0 bad. Checked 1 Rollback Requests found 0 bad. Checked 1 Rollback Confirms found 0 bad. Checked 0 Cancel Checked 0 Cancel Checked o status Checked o status Checked 1 Open Checked 1 Open Checked I Close Checked I Close Checked 1 Execute Checked 1 Execute Checked 0 Define Checked o Define Checked 0 Invoke Checked 0 Invoke Checked 0 Drop Checked 0 Drop Requests found 0 bad. Confirms found 0 bad. Requests found 0 bad. Confirms found 0 bad. Requests found 0 bad. Confirms found 0 bad. Requests found 0 bad. Confirms found 0 bad. Requests found 0 bad. Confirms found 0 bad. Requests found 0 bad. Confirms found 0 bad. Requests found 0 bad. Confirms found 0 bad. Requests found 0 bad. Confirms found 0 bad. Test RDA025 Complete.

RDA025 : Completion Status: PASSED. 


\section{APPENDIX D Sample Summary Report}

RDA/SQL Test suite

for RDA Server on rda

Mon Sep 23 16:42:14 1996

\begin{tabular}{|c|c|c|}
\hline PROGRAM & RESULT & Description of Test Case \\
\hline$-----n$ & ----- & ----------------------- \\
\hline $\mathrm{RDA001}$ & pass & singleArg, multipleArg missing -> 1 listofResultValues \\
\hline $\mathrm{RDA002}$ & pass & repetitioncount default is 1 \\
\hline $\mathrm{RDA003}$ & fail & repetitionCount $=10->10$ sQLDBExceptions, FETCH \\
\hline $\mathrm{RDA004}$ & pass & repetitioncount $=3->$ SQLDBExceptions, INSERT \\
\hline RDA005 & pass & 4 SQLDBLArgumentValues $->4$ sQLDBExceptions, SELECT \\
\hline RDA006 & pass & OPEN undeclared cursor name (syntax error) \\
\hline RDA007 & fail & cursor declaration survives CLOSE \\
\hline RDA008 & pass & cursor declaration survives ROLLBACK \\
\hline RDA009 & pass & cursor declaration survives COMMIT \\
\hline RDA010 & pass & null charset on R-Open $\rightarrow$ each charset has objId, CURSOR \\
\hline RDA011 & pass & null charset on R-Open $\rightarrow$ each charset has objId, SELECT \\
\hline RDA012 & pass & ConformanceLevel <1987>, objId 1.0 .9075 .0 \\
\hline $\mathrm{RDA013}$ & aborted & Conformancelevel <1989> without IEF, objId 1.0.9075.1.0.1 \\
\hline RDA014 & pass & ConformanceLevel <1989> with IEF, objId 1.0.9075.1.1.1 \\
\hline RDA015 & pass & Conformancelevel <1992> low, objId 1.0 .9075 .2 .0 \\
\hline RDA016 & fail & Table10.5b\&6a: DECLARE ArgSpec, OPEN ResSpec \\
\hline RDA017 & fail & Table10.5a\&6a: DECLARE ArgSpec override by OPEN, OPEN ResSpec \\
\hline RDA018 & fail & Table10.5a\&6b: OPEN Argspec, OPEN ResSpec \\
\hline RDA019 & fail & Table10.2a.2b.3b: DECLARE ArgSpec, FETCH overrides OPEN ResSpec \\
\hline $\mathrm{RDA0} 20$ & fail & Table10.2a.2b.3b: OPEN overrides DECLARE ArgSpec, FETCH overrides \\
\hline $\mathrm{RDA0} 21$ & fail & Table10.2a.2b.3b: OPEN ArgSpec, FETCH overrides OPEN ResSpec \\
\hline $\mathrm{RDAO} 22$ & pass & Table10.4a: SELECT ResSpec sent by client \\
\hline
\end{tabular}




\begin{tabular}{|c|c|c|}
\hline $\mathrm{RDA} 023$ & pass & Table10.4b: SELECT ResSpec ommitted by client \\
\hline RDA024 & pass & repetitioncount $=3 \rightarrow$ sQLDBExceptions, UPDATE \\
\hline $\mathrm{RDA025}$ & pass & 4 SQLDBLArgumentValues -> 4 sQLDBExceptions, DELETE \\
\hline RDA026 & pass & ERROR for ROLLBACK WORK in r-ExecuteDBL-RI \\
\hline RDA027 & pass & ERROR for COMMIT WORK in $r$-ExecuteDBL-RI \\
\hline $\mathrm{RDA} 028$ & pass & ERROR for INSERT with explicit sQLUsageMode = retrieval \\
\hline RDA029 & pass & ERROR for CURSOR UPDATE explicit sQLUsageMode = retrieval \\
\hline $\mathrm{RDA} 030$ & pass & ERROR for UPDATE WHERE explicit sQLUsageMode = retrieval \\
\hline RDA031 & pass & ERROR for DELETE WHERE explicit sQLUsageMode = retrieval \\
\hline RDA0́32 & pass & ERROR for CURSOR DELETE explicit sQLUsageMode = retrieval \\
\hline $\mathrm{RDA033}$ & pass & SIZING SQL statementText 4000 octets long \\
\hline $\mathrm{RDA} 034$ & pass & SIZING charset 16 elements long in Argspec \\
\hline RDA035 & pass & SIZING 100 entries in ArgSpec and ArgVal \\
\hline RDA036 & fail & Check that NULLs can be inserted and retrieved. \\
\hline $\mathrm{RDA} 037$ & pass & SIZING 100 entries of SQLDataTypeDescriptor in Resspec-RI \\
\hline $\mathrm{RDA038}$ & pass & SIZING repetitioncount $=64$ \\
\hline RDA039 & pass & SIZING 64 entries of SQLDBLArgumentValues in ArgVal \\
\hline RDA040 & pass & SIZING smallintType decimal precision 4 \\
\hline RDA041 & pass & SIZING charset 16 elements long in Resspec-RI \\
\hline $\mathrm{RDA042}$ & pass & SIZING integerType decimal precision 9 \\
\hline RDA043 & pass & SIZING 64 entries of ResultValueslist \\
\hline $\mathrm{RDA044}$ & pass & SIZING 100 entries of SQLValue in SQLDBLResultValues \\
\hline $\mathrm{RDA045}$ & pass & SIZING colName 18 characters long \\
\hline RDA046 & pass & SIZING length (characters) 240 (Including null terminator) \\
\hline $\mathrm{RDA} 047$ & pass & SIZING numericType $(15,0)$ (cut to $(2 * \star 31-1)$ ) \\
\hline $\mathrm{RDA} 048$ & fail & SIZING decimalType precision 8 scale 8 \\
\hline RDA049 & missing & SIZING integerType decimal precision 9 \\
\hline
\end{tabular}


NISTIR 5725

User's Guide for RDA/SQL Validation Tests (Version 1.0)

Kevin Brady

Joan Sullivan

September 1996

U.S. DEPARTMENT OF COMMERCE

Technology Administration

National Institute of Standards and Technology

Gaithersburg, MD 20899 
User's Guide for the RDA/SQL Validation Tests (Version 1.0)

Kevin Brady

Joan Sullivan

September 1996

ABSTRACT: The RDA/SQL Validation Tests, developed by NIST, consist of a set of $C$ programs designed to test an RDA/SQL server for conformance to the international standards for Remote Database Access with an SQL Specialization. Testing is limited to the RDA Basic Application Context/Immediate Execution profile defined by the NIST OIW Stable Agreements for OSI Protocols. The validation tests use software tools provided in the public domain Iso Development Environment (ISODE) and has been operating at NIST on TCP/IP networks using the Internet Engineering Task Force (IETF) specification RFC 1006.

KEYWORDS: conformance testing; database standards; interoperability; remote database access; RDA; SQL; RFC 1006; ISODE; testing of software; validation of software 



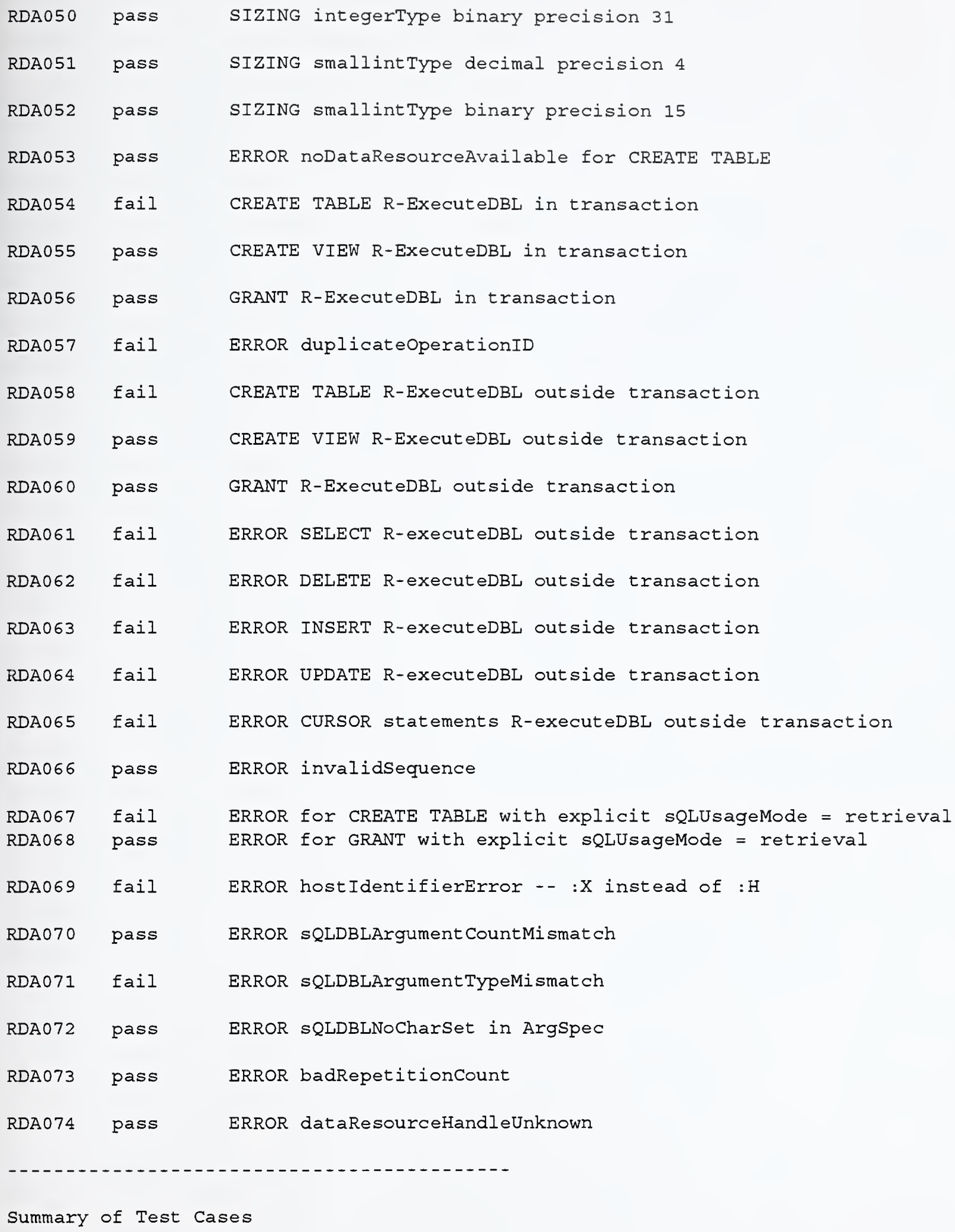


tests passed: 0051

tests failed: 0021

tests missing: 0001

tests aborted: 0001

TOTAL tests: $\quad 0074$ 

